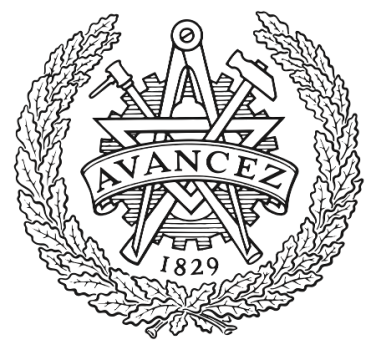

CHALMERS

UNIVERSITY OF TECHNOLOGY

\title{
Transmitter Beam Selection in Millimeter-Wave MIMO with In-Band Position-Aiding
}

Downloaded from: https://research.chalmers.se, 2023-04-26 14:29 UTC

Citation for the original published paper (version of record):

Garcia, G., Seco-Granados, G., Karipidis, E. et al (2018). Transmitter Beam Selection in Millimeter-Wave MIMO with In-Band Position-Aiding. IEEE Transactions on Wireless Communications, 17(9): 6082-6092. http://dx.doi.org/10.1109/TWC.2018.2854583

N.B. When citing this work, cite the original published paper.

C2018 IEEE. Personal use of this material is permitted.

However, permission to reprint/republish this material for advertising or promotional purposes 


\title{
Transmitter Beam Selection in Millimeter-wave MIMO with In-Band Position-Aiding
}

\author{
Gabriel E. Garcia, Gonzalo Seco-Granados, Member, IEEE, Eleftherios Karipidis, Member, IEEE \\ and Henk Wymeersch, Member, IEEE
}

\begin{abstract}
Emerging wireless communication systems will be characterized by a tight coupling between communication and positioning. This is particularly apparent in millimeter-wave (mm-wave) communications, where devices use a large number of antennas and the propagation is well described by geometric channel models. For mm-wave communications, initial access, consisting in the beam selection and alignment of two devices, is challenging and time-consuming in the absence of location information. Conversely, accurate positioning relies on highquality communication links with proper beam alignment. This paper studies this interaction and proposes a new position-aided transmitter beam selection protocol, which considers the problem of joint communication and positioning in scenarios with direct line-of-sight and scattering. Simulation results show significant reductions in latency with respect to a standard protocol.
\end{abstract}

\section{INTRODUCTION}

M ILLIMETER-WAVE (mm-wave) communications have recently gained attention for the development of highspeed wireless networks. Mm-wave systems operate at frequencies between 30 to $300 \mathrm{GHz}$ with large available bandwidths. Combined with multiple-input-multiple-output (MIMO), using a large number of antennas, mm-wave can provide high data rates to users through dense spatial multiplexing [1]-[5]. Hence, mm-wave MIMO is considered a key enabler for emerging communication systems, e.g., 5G or IEEE WiGig [6], to deliver throughputs on the order of multi-Gbps for a range of applications from wearables [7] to automotive [8]. However, mm-wave communications face a number of challenges, in particular severe path-loss at these high frequencies. As a solution, system designers improve the link budget through highly directional links involving sophisticated beamforming (BF) at the transmitter and/or receiver [9]-[11], relying on the knowledge of the mm-wave MIMO propagation channel.

Given the propagation behaviour of mm-wave where only the line-of-sight (LOS) path and a few dominant multipath components contribute to the received power, stochastic geometrical channel models have become an attractive approach

G. E. Garcia, and H. Wymeersch are with the Department of Electrical Engineering, Chalmers University of Technology, Gothenburg, Sweden, e-mails: \{ggarcia,henkw\} @chalmers.se. G. Seco-Granados is with the Department of Telecommunications and Systems Engineering, Universitat Autònoma de Barcelona, Barcelona, Spain, e-mail: gonzalo.seco@uab.cat. E. Karipidis is with Ericsson Research, Stockholm, Sweden, e-mail: eleftherios.karipidis@ericsson.com. This research was supported, in part, by the European Research Council, under Grant No. 258418 (COOPNET), the EU project HIGHTS (High precision positioning for cooperative ITS applications) MG-3.5a-2014-636537, and the R\&D Project of Spanish Ministry of Economy and Competitiveness under Grant TEC2014-53656-R. to characterize the channel with few parameters. These models relate the propagation to the geometry of the operating environment, thus creating an explicit interplay between the communication channel and the positions of the transmitter, receiver, and reflectors [12]-[16]. This interplay becomes apparent during the initial access procedure, where two devices, a transmitter and a receiver, here termed D1 and D2, aim to establish a connection by achieving beam alignment. This consists in finding a pair of transmit and receive beams to reach a required signal-to-noise ratio (SNR) for the link. The initial access is a fundamental procedure pertinent to pratical communication systems that preceeds data transmission. The procedure enables the establishment of a sufficient link budget, typically for the purpose of acquiring time and frequency synchronization, and receiving control information, such as scheduling grants and reference signals. The initial access problem is solved with the purpose of discovering new propagation paths and discard paths with low power as well as to serve as a first step towards downlink training, feedback and downlink/uplink communication. From the communications perspective, this is achieved by a dedicated protocol that searches across the angle-of-arrival (AOA) and angle-ofdeparture (AOD) space. Both AOA and AOD can be related to the location of D1 and D2, thus presenting an opportunity to exploit location information. From the positioning perspective, estimating the position (two-dimensional or three-dimensional) and orientation of a device through exchange of mm-wave signals requires the establishment of a communication link. Hence, the communication and positioning problems are coupled, indicating that a joint solution strategy may yield better performance.

Conventional beam selection protocols do not consider the positioning aspect explicitly. For instance, the authors in [9], [17]-[20] designed BF protocols based on discretized iterative beam codebooks, while in [11] the use of simultaneous beams through beam coding is introduced. In [15], [21], the authors developed hierarchical multi-resolution codebooks: in [15], codebooks are based on hybrid analog/digital precoding and proposed low-overhead channel estimation algorithms, while in [21] the codebook allows for beam overlapping for channel estimation purposes. In [22], the initial access problem is tackled by means of scanning and signaling procedures, while in [23] the authors propose a strategy for transmitting reference signals using pre-designed codebooks for device discovery, and in [24], prioritized beam ordering strategies are presented. These protocols involve a time-consuming search over different AOA/AOD pairs in order to determine directions in which 
to point the beams. On the other hand, contributions in the area of positioning generally ignore the initial access aspect. For instance, the authors in [13], [25]-[28] present direction-ofarrival and location estimation algorithms, but do not provide initial access protocols. Similarly, [29] exploits mm-wave and MIMO features along with BF to provide sufficient conditions on the identifiability of the position and orientation for a device in a LOS scenario but no protocols for the initial access are included. Works that combine positioning with initial access include [30]-[33]: [30] proposes a beam alignment method for fixed-position network nodes in mm-wave backhaul systems aided with position information obtained using high-sensitivity displacement sensors in each node. In [31], beam training is presented exploiting a database linked to the geographical position of the users. In [32] location information is harnessed for fast channel estimation in a vehicular context. In [33], beam alignment is proposed with the use of position information obtained from the on-board train system. What is common in [30]-[33] is that position information is obtained out-ofband, not from the mm-wave signal itself. In the context of beam tracking (i.e., once the initial access has been solved) in-band information has been harnessed, in the form of either AOD or/and AOA [34]-[36]: authors in [35] propose an estimator for the AOD and channel information under Gaussian AOD dynamics, but no protocol is presented; in [34], AOA estimation is introduced based on the geometry of the antenna array and the transmitting beam pattern, not including position information; in [36], state-space models for the AOD and AOA are inferred aided with channel-aided information rather than position information.

In this paper, a novel in-band positioning-aided transmitter beam selection protocol is proposed, with the aim of reducing the set-up time of the initial access procedure for communication in the presence of a LOS path and unknown scatterer locations. In order to gain insight into the fundamental achievable performance, we determine the evolution of the Fisher information of the D2 position and orientation as new beams are utilized, feeding back this location information to D1 in order to adapt the beams. Both discrete and continuous codebooks are considered. The new protocol is evaluated through simulations, considering as performance metrics the set-up time, signal-to-noise (SNR) ratio, and the position and orientation error bounds after protocol completion. We observe that the position-aided protocol is significantly faster than a conventional protocol based on discretized beam codebooks, with little or no SNR penalty, and can additionally determine the position or orientation of D2. In addition, we find that standard discrete codebooks achieve similar performance to more complex codebooks, indicating that the proposed protocol can be implemented with standard mm-wave communication technologies.

The remainder of the paper is structured as follows. Section II presents the communication model and performance metrics. In Section III, the conventional protocol description, operation and performance are described. Then, in Section IV the joint positioning and transmitter beam selection protocol, its operation and performance are introduced. Finally, numerical results are given in Section $\mathrm{V}$, followed by the conclusions in
Section VI.

\section{SySTEM MODEL}

\section{A. Geometric Model}

We consider a MIMO mm-wave system consisting of a transmitting device D1 with $N_{t}$ antennas and beamforming capabilities, and a receiving device D2 with $N_{r}$ antennas. The 2-dimensional locations ${ }^{1}$ of D1 and D2 are denoted by $\mathbf{p}=\left[p_{\mathrm{x}}, p_{\mathrm{y}}\right]^{\mathrm{T}} \in \mathbb{R}^{2}$ and $\mathbf{q}=\left[q_{\mathrm{x}}, q_{\mathrm{y}}\right]^{\mathrm{T}} \in \mathbb{R}^{2}$, respectively, and let $\alpha \in[0,2 \pi)$ be the angle of rotation of the D2 antenna array with respect to the horizontal axis. These parameters in turn imply an AOD $\theta_{\mathrm{tx}, 0}$ and an AOA $\theta_{\mathrm{rx}, 0}$, as depicted in Figure 1. Note that under our definitions, $\cos \left(\theta_{\mathrm{tx}, 0}\right)=$ $\left(p_{\mathrm{x}}-q_{x}\right) /\|\mathbf{q}-\mathbf{p}\|$, and $\alpha=\pi+\theta_{\mathrm{tx}, 0}-\theta_{\mathrm{rx}, 0}$. We also introduce the LOS propagation delay as between D2 and D1 as $\tau_{0}=\|\mathbf{q}-\mathbf{p}\| / c$, where $c$ is the speed of light. We assume that $\mathbf{q}$ is a known reference point. It is easy to show that the knowledge of $\boldsymbol{\beta}=[\mathbf{p}, \alpha]^{\mathrm{T}}$ is equivalent to the knowledge of $\left[\tau_{0}, \theta_{\mathrm{tx}, 0}, \theta_{\mathrm{rx}, 0}\right]$. The environment can also contain scatterers, here modeled as points, with locations $\mathbf{s}_{k}, k \geq 1$, for which we introduce $\tau_{k}=\left\|\mathbf{q}-\mathbf{s}_{k}\right\| / c+\left\|\mathbf{s}_{k}-\mathbf{p}\right\| / c$, as well as the AOD $\theta_{\mathrm{tx}, k}$ and AOA $\theta_{\mathrm{rx}, k}$, as shown in Figure 1.

Furthermore, we consider that the device D1 transmits signals at a carrier frequency $f_{c}$ (or equivalently wavelength $\lambda=c / f_{c}$, where $c$ is the speed of light) and with bandwidth $B$. We employ a narrowband model ${ }^{2}$ where the $N_{r} \times N_{t}$ channel matrix is given by [37], [38]

$$
\mathbf{H}(t)=\sum_{k=0}^{K-1} \underbrace{\sqrt{N_{t} N_{r}} h_{k} \mathbf{a}_{\mathrm{rx}}\left(\theta_{\mathrm{rx}, k}\right) \mathbf{a}_{\mathrm{tx}}^{\mathrm{H}}\left(\theta_{\mathrm{tx}, k}\right)}_{=\mathbf{H}_{k}} \delta\left(t-\tau_{k}\right),
$$

in which $h_{k}$ is the complex gain of the $k$-th path, $\mathbf{a}_{\mathrm{tx}}\left(\theta_{\mathrm{tx}, k}\right) \in$ $\mathbb{C}^{N_{t}}$ and $\mathbf{a}_{\mathrm{rx}}\left(\theta_{\mathrm{rx}, k}\right) \in \mathbb{C}^{N_{r}}$ are the normalized antenna steering and response vectors associated with the $k$-th path. When two paths are unresolvable, they are combined into a single path by adding their complex gains. Consider paths $a$ and $b$, with AOAs $\theta_{r, a}$, and $\theta_{r, b}$; delays $\tau_{a}$, and $\tau_{b}$, respectively. Paths $a$ and $b$ are considered unresolvable in time and angle, when $\left|\tau_{a}-\tau_{b}\right| \leq 1 / B$ and $N_{r} \lambda\left|\sin \left(\theta_{r, a}\right)-\sin \left(\theta_{r, b}\right)\right| \leq d$ [39]. Without loss of generality, our focus will be on uniform linear arrays $^{3}$ (ULA), for which

$$
\begin{aligned}
{\left[\mathbf{a}_{\mathrm{tx}}\left(\theta_{\mathrm{tx}}\right)\right]_{l=0}^{N_{t}-1} } & =\frac{1}{\sqrt{N_{t}}} \exp \left(j \frac{2 \pi l d}{\lambda} \sin \theta_{\mathrm{tx}}\right) \\
{\left[\mathbf{a}_{\mathrm{rx}}\left(\theta_{\mathrm{rx}}\right)\right]_{l=0}^{N_{r}-1} } & =\frac{1}{\sqrt{N_{r}}} \exp \left(j \frac{2 \pi l d}{\lambda} \sin \theta_{\mathrm{rx}}\right)
\end{aligned}
$$

where $d$ is the antenna spacing.

\footnotetext{
${ }^{1}$ A 2-dimensional model is assumed for simplicity. However, the proposed protocols can be extended to 3-dimensional scenarios with 2-dimensional antenna arrays.

${ }^{2}$ The narrowband assumption imposes the constraints that (i) there is no beam squint; and (ii) $\max \left(N_{t}, N_{r}\right) d \ll c / B$ where $d$ denotes the distance between the antenna elements.

${ }^{3}$ The underlying idea of the proposed protocol is applicable to any array geometry.
} 


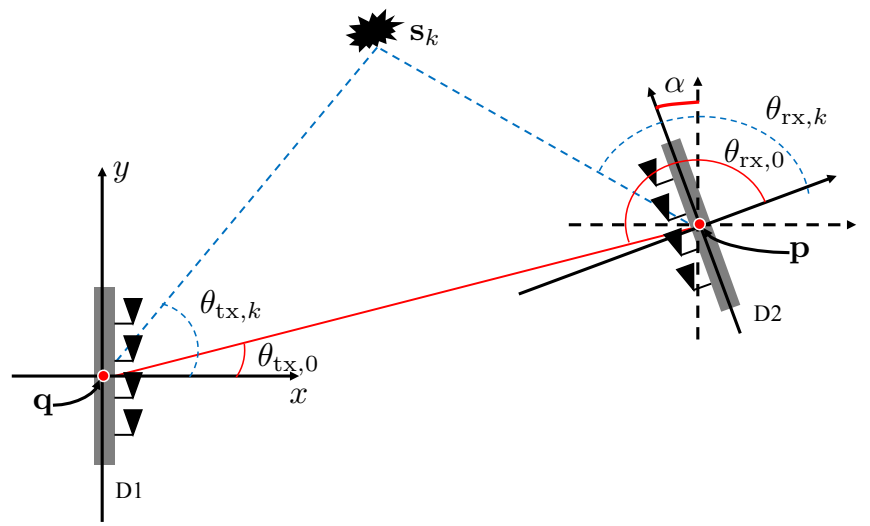

Figure 1. Two-dimensional MIMO system model with a D1 with known position and orientation, and a D2 with unknown position (p) and orientation $(\alpha)$. The AODs $\theta_{\mathrm{tx}, k}$ and AOAs $\theta_{\mathrm{rx}, k}$ are also indicated.

\section{B. Training Model}

1) Transmitter With Analog Beamforming: We assume the use of analog beamforming, implemented with phase shifters and combined with antenna selection. The transmitter D1 can sequentially send training sequences (TS) using beams pointed towards in different directions, leading to a signal model $\mathbf{f}_{m} x(t)$ in which $x(t)=\sum_{n=1}^{N} g_{n} p(t-n / B)$, where $p(t)$ is a unit-energy transmit pulse (e.g., root-raised cosine), $N$ denotes the number of symbols, and $g_{n}$ are known training symbols with $\mathbb{E}\left\{\left|g_{n}\right|^{2}\right\}=E_{s}$, and

$$
\begin{aligned}
& \mathbf{f}_{m}= \\
& \frac{1}{\sqrt{N_{t}^{\prime}}}\left[\mathbf{0}_{\left.\mid \frac{N_{t}-N_{t}^{\prime}}{2}\right\rfloor} e^{j \phi_{0}} \ldots e^{j \phi_{N_{t}^{\prime}-1}} \mathbf{0}_{\left\lceil\frac{N_{t}-N_{t}^{\prime}}{2}\right\rceil}\right]^{\mathrm{T}},
\end{aligned}
$$

where $\mathbf{0}_{r}$ is defined as a null vector of size $r$ and $N_{t}^{\prime} \leq N_{t}$ indicates the number of active contiguous antennas [40] used to control the beam widths at the expense of the beam gain. Special cases include (i) the use of 90-degree phase resolution to minimize power consumption [9]

$$
\phi_{i} \in\{0, \pi, \pi / 2,-\pi / 2\}
$$

and (ii) unconstrained directional

$$
\phi_{i}=e^{j \frac{2 \pi d i}{\lambda} \sin \theta_{m}},
$$

where $\theta_{m}$ is the direction of the beam, chosen from a given set $\Theta$. The design parameters of the beam patterns consist of the maximum gain direction $\theta_{\max }$ and the half-power beamwidth angle, $\theta_{\mathrm{HPBW}}$, which is the angle where the square magnitude of the radiation pattern decreases by $50 \%$ with respect to its maximum value, and depends on the type of antenna and operating frequency, among other parameters. For beams based on (5) or (6), both $\theta_{\max }$ and $\theta_{\mathrm{HPBW}}$ can be calculated and tabulated [41].

2) Idealized Receiver: For each transmitted beam, the receiver observes the following complex baseband signal:

$$
\mathbf{y}(t)=\sum_{k=0}^{K-1} \mathbf{H}_{k} \mathbf{f}_{m} x\left(t-\tau_{k}\right)+\mathbf{n}(t)
$$

where $\mathbf{n}(t) \in \mathbb{C}^{N_{r}}$ is a Gaussian noise vector with zero mean and two-sided power spectral density $N_{0} / 2$. We will consider an idealized receiver D2, which samples the entire signal $\mathbf{y}(t)$ and is synchronized to D1 [42]. While such a receiver may be impractical, it allows us to understand the ultimate performance of position-aided protocols and can thus serve as a benchmark for different receiver structures with analog beamforming as well as low-complexity algorithms.

\section{Performance Metrics}

The performance of initial access protocols can be assesed on their delay and its ability to discover the dominant path. A reduced delay leads to a two-fold benefit: (i) reduced overhead of the initial access procedure, and thus, more time for data communication, leading to increased throughput; (ii) the ability to deal with higher mobility, since paths can be discovered faster [43]. The beam selection protocol works in an iterative manner, where each iteration $i \geq 1$ involves selecting a number of active antennas $N_{t}^{(i)}<N_{t}$ and a number of beams $M_{t}^{(i)}$. The objective of protocol is to quickly determine a beamforming vector $\mathbf{f}_{\text {sel }}$ resulting in high SNR. The relevant performance metrics are thus SNR, number of transactions, and positioning quality.

1) SNR: The selection of $\mathbf{f}_{\text {sel }}$ intends to maximize the SNR, which assuming the $K$ paths are both resolvable in the time and/or angular spaces is then defined as

$$
\mathrm{SNR} \triangleq \sum_{k=0}^{K-1} \frac{N_{t} N_{r} E_{s}}{N_{0}}\left|h_{k}\right|^{2}\left\|\mathbf{a}_{\mathrm{tx}}^{\mathrm{H}}\left(\theta_{\mathrm{tx}, k}\right) \mathbf{f}_{\mathrm{sel}}\right\|
$$

2) Number of transactions: Considering an iterative beam selection protocol, total beam selection time can be broken down for each iteration $i \geq 1$ as follows: (i) training stage during which D1 sends $M_{t}^{(i)}$ training sequences; (ii) feedback stage, during which D2 reports back to D1; (iii) mapping stage, during which D1 informs D2 about the number of required transmit beam patterns $M_{t}^{(i+1)}$; and a one time (iv) acknowledgment after which high-rate data communication can start. The total number of transactions $N_{\text {trans }}$ can be quantified as $N_{\text {trans }}=\sum_{i=1}^{I}\left(M_{t}^{(i)}+2\right)+1$. Note that when the mapping is agreed a priori and feedback messages are neglected, we find that

$$
N_{\text {trans }} \approx \sum_{i=1}^{I} M_{t}^{(i)}
$$

Given the idealized receiver D2, we consider that each transaction takes the same absolute time for each of the presented beam selection protocols for comparison fairness.

3) Positioning quality: We consider the expected positioning and orientation errors, given by

$$
\mathbb{E}\left\{\|\mathbf{p}-\hat{\mathbf{p}}\|^{2}\right\}
$$

and

$$
\mathbb{E}\left\{\|\alpha-\hat{\alpha}\|^{2}\right\},
$$


where $\hat{\mathbf{p}}$ and $\hat{\alpha}$ denote the estimated position and angle of rotation for the $\mathrm{D} 2$, respectively, obtained from the sequence of received signals of the form (7).

\section{Conventional Beam Selection}

A beam selection protocol with the goal of minimizing the beamforming set-up time and mitigate the high pathloss has been adopted by the IEEE 802.15 .3c standard as an optional functionality [9]. This iterative protocol relies on a multi-level beam tree search starting from lower resolution beams that cover large angular range per beam moving towards higher resolution beams covering a smaller angular range. Other protocols have been considered in the literature [10], [17], [19], [20] . Here, we describe a general beam selection protocol for D1, not exploiting or requiring any position information.

\section{A. General Protocol Operation}

The iterative protocol selects a number of active antennas and a number of beams at each iteration $i \geq 1$. In particular, at iteration $i$, D1 selects $M_{t}^{(i)}$ beams with associated beamforming vectors

$$
\mathbf{F}^{(i)}=\left\{\mathbf{f}_{1}^{(i)}, \ldots, \mathbf{f}_{M_{t}^{(i)}}^{(i)}\right\}
$$

to be used with $N_{t}^{(i)} \leq N_{t}$ selected active antennas. The protocol makes use of a finite codebook from which beams can be selected for each value of $N_{t}^{(i)}$. D1 transmits a reference signal $x(t)$ for each of the $M_{t}^{(i)}$ beams. Through suitable signal processing, D2 measures the reference signal received power (RSRP) $P_{m}^{(i)}$ for each of the $m=1, \ldots, M_{t}^{(i)}$ transmitted beams, and gathers them in the vector $\mathbf{P}^{(i)} \in$ $\mathbb{R}^{M_{t}^{(i)}}$. The selection of the beams at each iteration $i$ is dependent on the previous beam selection $\mathbf{F}^{(i-1)}$ and on the RSRPs $\mathbf{P}^{(i-1)}$ transmitted as feedback from D2 to D1. The mapping $\mathbf{F}^{(i)}=f_{\text {map }}\left(\mathbf{F}^{(i-1)}, \mathbf{P}^{(i-1)}\right)$ depends on the specific codebook employed. The protocol is summarized as pseudocode in Algorithm 1.

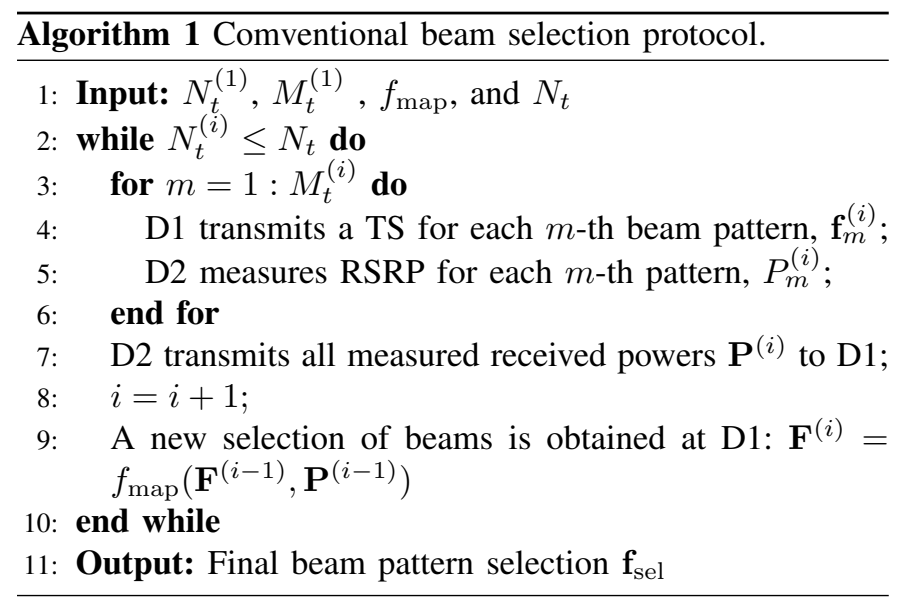

\section{B. Protocol-specific Performance}

We now present the evaluation of the performance metrics in Section II.C for the RSRP protocol.

1) SNR: Upon completion of the protocol, at iteration $I$, corresponding to $N_{t}^{(I)}=N_{t}$, the beam with the highest RSRP is selected $\mathbf{f}_{\text {sel }}=\mathbf{f}_{m^{*}}^{(I)}$ by solving

$$
m^{*}=\arg \max _{m} P_{m}^{(I)},
$$

and the SNR is evaluated according to (8).

2) Number of transactions: Depending on the codebook design, which is known both at D1 and D2, different implementations of the protocol can be designed by means of the mapping $\mathbf{F}^{(i)}=f_{\text {map }}\left(\mathbf{F}^{(i-1)}, \mathbf{P}^{(i-1)}\right)$, e.g, [9], [32]. A simple mapping would involve one iteration with $M_{t}=N_{t}$ narrow beams, leading to a number of transactions $N_{\text {trans }}^{\text {conv }} \approx N_{t}$ [24]. A reduction in delay can be achieved through a multi-level beam search from broad to directive beams, noting that the half-power beamwidth scales roughly as $1 / N_{t}^{(i)}$ [41], so that a beam with $N_{t}^{(i-1)}$ antennas can be covered with $M_{t}^{(i)} \leq 3$ beams with $N_{t}^{(i)}=2 N_{t}^{(i-1)}$ antennas. This leads to $I=\log _{2}\left(N_{t}\right)$ and thus $N_{\text {trans }}^{\text {conv }} \approx 3 \log _{2}\left(N_{t}\right)$.

3) Positioning quality: The protocol does not provide any positioning information.

\section{Proposed Joint Positioning And BeAm SELECTION}

In this Section, we introduce the proposed iterative positionbased beam selection protocol. The protocol aims to minimize the set-up time and mitigate the high path-loss using D2 position information as proxy for the optimal beamforming direction of the transmit beams. Before we describe the proposed protocol, followed by its performance and implementation details, we first briefly detail some properties of mm-wave positioning.

\section{A. Performance of Mm-wave Positioning}

D2 can perform estimation of its position and orientation (represented by $\boldsymbol{\beta}$ ) based on the received waveforms from D1. The quality of such estimation can be assessed through the Fisher information matrix (FIM) [44]. Given the statistics of a waveform $\mathbf{y}(t)$ of the form (7) and an unknown vector parameter

$$
\boldsymbol{\eta}=\left[\tau_{0}, \boldsymbol{\theta}_{0}^{\mathrm{T}}, \mathbf{h}_{0}^{\mathrm{T}}, \ldots, \tau_{K-1}, \boldsymbol{\theta}_{K-1}^{\mathrm{T}}, \mathbf{h}_{K-1}^{\mathrm{T}}\right]^{\mathrm{T}},
$$

where $\boldsymbol{\theta}_{k}=\left[\theta_{\mathrm{tx}, k}, \theta_{\mathrm{rx}, k}\right]^{\mathrm{T}}, \quad \mathbf{h}_{k}=\left[h_{R, k}, h_{I, k}\right]=$ $\left[\Re\left\{h_{k}\right\}, \Im\left\{h_{k}\right\}\right]$, the FIM associated with a single beam, $\mathbf{J}_{\boldsymbol{\eta}}^{\text {(beam) }}$, is a $5 K \times 5 K$ matrix, whose expression and its submatrices are provided in (22), and (21) in the Appendix.

While each $\mathbf{y}(t)$ corresponds to a single transmit beam, the FIM for multiple beams is simply the sum of the corresponding FIMs, due to the additive nature of Fisher information. For each iteration $i$ of a beam selection protocol, we can thus compute the FIM associated with the $m$-th beam, say, $\mathbf{J}_{\boldsymbol{\eta}}^{(i, m)}$. The total FIM after $i$ iterations can then be expressed as 


$$
\mathbf{J}_{\boldsymbol{\eta}}^{(i)}=\sum_{l=1}^{i} \sum_{m=1}^{M_{t}^{(l)}} \mathbf{J}_{\boldsymbol{\eta}}^{(l, m)} .
$$

Since there is an injective relation between $\boldsymbol{\eta}$ and ${ }^{4}$

$$
\boldsymbol{\eta}^{\prime}=\left[\boldsymbol{\beta}^{\mathrm{T}}, \mathbf{h}_{0}^{\mathrm{T}}, \mathbf{s}_{1}^{\mathrm{T}}, \mathbf{h}_{1}^{\mathrm{T}}, \ldots, \mathbf{s}_{K-1}^{\mathrm{T}}, \mathbf{h}_{K-1}^{\mathrm{T}}\right]^{\mathrm{T}},
$$

we can also determine the FIM of $\mathbf{J}_{\eta^{\prime}}^{(i)}$ as $\mathbf{J}_{\eta^{\prime}}^{(i)}=\mathbf{T}^{\mathrm{T}} \mathbf{J}_{\boldsymbol{\eta}}^{(i)} \mathbf{T}$, where $\mathbf{T}$ is the Jacobian matrix associated with the transformation from $\boldsymbol{\eta}$ to $\boldsymbol{\eta}^{\prime}$, that is, $T_{i j}=\partial \eta_{i} / \partial \eta_{j}^{\prime}$.

Finally, the inverse of the FIM can be related to the mean squared error (MSE) of unbiased estimators of $\boldsymbol{\eta}^{\prime}$ [44]:

$$
\mathbb{E}_{\mathbf{y} \mid \boldsymbol{\eta}^{\prime}}\left[\left(\hat{\boldsymbol{\eta}}^{\prime}-\boldsymbol{\eta}\right)\left(\hat{\boldsymbol{\eta}}^{\prime}-\boldsymbol{\eta}\right)^{\mathrm{T}}\right] \succeq\left[\mathbf{J}_{\boldsymbol{\eta}^{\prime}}^{(i)}\right]^{-1} .
$$

From this relationship, we can immediately derive the socalled position error bound (PEB) and rotation error bound (REB) as

$$
\begin{aligned}
\operatorname{PEB}^{(i)} & =\sqrt{\operatorname{tr}\left\{\left[\mathbf{J}_{\boldsymbol{\eta}^{\prime}}^{(i)}\right]_{1: 2,1: 2}^{-1}\right\}} \\
& \leq \sqrt{\mathbb{E}\left\{\|\mathbf{p}-\hat{\mathbf{p}}\|^{2}\right\}}
\end{aligned}
$$

and

$$
\begin{aligned}
\operatorname{REB}^{(i)} & =\sqrt{\left[\mathbf{J}_{\boldsymbol{\eta}^{\prime}}^{(i)}\right]_{3,3}^{-1}} \\
& \leq \sqrt{\mathbb{E}\left\{\|\alpha-\hat{\alpha}\|^{2}\right\}}
\end{aligned}
$$

where $[\cdot]_{1: 2,1: 2}^{-1}$ denotes the $2 \times 2$ upper left submatrix of the inverse of the argument, and $[\cdot]_{3,3}^{-1}$ denotes the third diagonal element of the inverse of the argument.

Remarks :

- We note that in contrast to conventional range-based positioning, the use of multiple antennas at both devices allows for the determination of both the position and the orientation of D2 using signals from a single reference device D1.

- The FIM and corresponding PEB and REB are valid, irrespective of the subsequent processing at the receiver. Such processing can include analog beamforming as well as low-complexity estimation and detection algorithms.

- Given the geometric model, the FIM does not account for path resolvability in time and angle spaces. When two paths are unresolvable, they are to be combined into a single path by adding the complex channel gains, prior to computation of the Fisher information. Unresolvability conditions were previously introduced in Section II.A.

\section{B. General Protocol Operation}

From the above FIM analysis, it is apparent that D2 can not only compute the received powers for each beam, but also harness them to compute its position and orientation $\boldsymbol{\beta}=[\mathbf{p}, \alpha]^{\mathrm{T}}$. We will denote the aggregated waveforms at iteration $i$ by $\mathbf{y}^{(i)}$, and the collection of $\mathbf{y}^{(i)}$ up to iteration

\footnotetext{
${ }^{4}$ In a practical implementation a priori information on the number of scatterers is not required [45].
}

$i$ by $\mathbf{y}^{(1: i)}$. Our idealized receiver D2 can thus be equipped with an estimator, which can determine an estimate of $\boldsymbol{\beta}$ from $\mathbf{y}^{(1: i)}$ at the end of each iteration $i$, operating close to the fundamental performance bounds (16)-(17). Considering a Gaussian approximation of the position and orientation error, we can describe the estimate by a mean $\hat{\boldsymbol{\beta}}^{(i)}$ and a covariance matrix $\boldsymbol{\Sigma}_{\boldsymbol{\beta}}^{(i)}$. This information can be fed back to D1. The protocol then operates according to Algorithm 2. Since D1 has more information about D2 than in the conventional algorithm, a more intelligent mapping function can be designed, as will be described in Section IV.C. In addition, both D2 and D1 have knowledge of D2's position and orientation.

\section{Remark:}

- The idealized receiver assumption serves to understand the fundamentals behind the use of position information in the initial access procedure and thus provides a bound for any real receiver. We expect real receivers equipped with practical estimators to operate close to the performance bounds at medium to high SNRs.

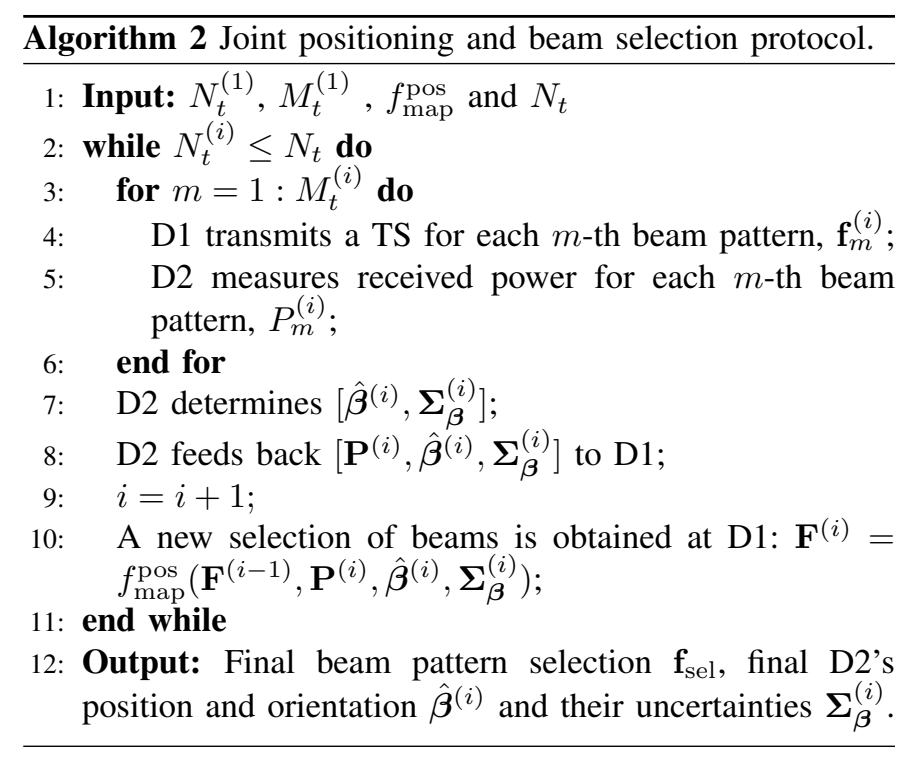

\section{Protocol-specific Performance}

We now present the evaluation of the performance metrics in Section II.C for the protocol described by Algorithm 2.

1) SNR: As for the conventional protocol, the SNR is computed using (8), based on the final selected beam.

2) Number of transactions: Different implementations depending on the mapping function $f_{\text {map }}^{\text {pos }}\left(\mathbf{F}^{(i-1)}, \mathbf{P}^{(i)}, \hat{\boldsymbol{\beta}}^{(i)}, \boldsymbol{\Sigma}_{\boldsymbol{\beta}}^{(i)}\right)$ can be designed to reduce the number of transactions. In particular, when D1 has knowledge of the AOD, it can select an appropriate number of active antennas and beams. More specifically, D1 can process $\hat{\boldsymbol{\beta}}^{(i)}, \boldsymbol{\Sigma}_{\boldsymbol{\beta}}^{(i)}$ to compute an AOD estimate $\hat{\theta}_{\mathrm{tx}, 0}^{(i)}$ and the AOD standard deviation, denoted as $\sigma_{\mathrm{tx}, 0}^{(i)}$. A conventional hierarchical protocol with $N_{t}^{(i)}=2 N_{t}^{(i-1)}$ and $M_{t}^{(i)}=3$ can be used whenever the AOD uncertainty is large, i.e., $2 \sigma_{\mathrm{tx}, 0}^{(i)} \geq 3 \theta_{\mathrm{HPBW}}\left(2 N_{t}^{(i-1)}, \hat{\theta}_{\mathrm{tx}, 0}^{(i)}\right)$. On the other hand, 


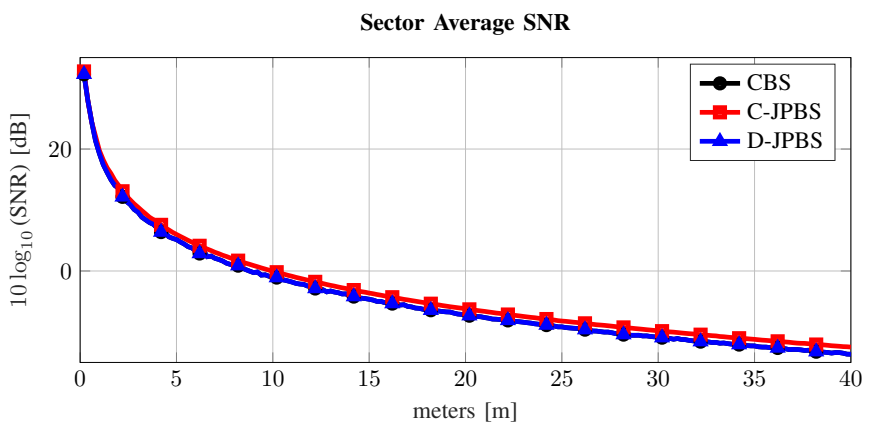

Figure 2. Average SNR as a function of distance to D2.

if $2 \sigma_{\mathrm{tx}, 0}^{(i)}<3 \theta_{\mathrm{HPBW}}\left(2 N_{t}^{(i-1)}, \hat{\theta}_{\mathrm{tx}, 0}^{(i)}\right)$, the number of transactions can be reduced by using more than $2 N_{t}^{(i)}$ active antennas with $M_{t}^{(i)}=3$. In the latter case, we propose to set $N_{t}^{(i)}$ according to

$$
\begin{aligned}
\operatorname{maximize} & N_{t}^{(i)} \\
\text { subject to } & N_{t}^{(i)} \leq N_{t} \\
& 3 \theta_{\mathrm{HPBW}}\left(N_{t}^{(i)}, \hat{\theta}_{\mathrm{tx}, 0}^{(i)}\right) \geq 2 \sigma_{\mathrm{tx}, 0}^{(i)},
\end{aligned}
$$

and transmit 3 beams covering the AOD region $\hat{\theta}_{\mathrm{tx}, 0}^{(i)} \pm$ $\sigma_{\mathrm{tx}, 0}^{(i)}$. Such beams can also be optimized to minimize the future expected uncertainty, as detailed in Appendix B. We thus expect that in cases when $\boldsymbol{\beta}$ can be accurately estimated with few antennas, then the number of transactions fulfills $N_{\text {trans }}^{\text {pos }} \ll N_{\text {trans }}^{\text {conv }}$, and $N_{\text {trans }}^{\text {pos }} \approx N_{\text {trans }}^{\text {conv }}$ otherwise. From [29], [46], it is known that good estimates of $\boldsymbol{\beta}$ are possible when enough beams are transmitted pointing roughly in the direction of $\mathrm{D} 2$, and the received SNR associated with those beams is sufficiently high. Consequently, we expect $N_{\text {trans }}^{\text {pos }} \ll N_{\text {trans }}^{\text {conv }}$ for D2 locations close to D1.

3) Positioning quality: The proposed protocol can be assessed in terms of position and orientation errors , which can be predicted through the FIM using (16)-(17).

\section{Simulation Results}

\section{A. Simulation Setup}

We consider a $40 \mathrm{~m} \times 40 \mathrm{~m}$ area where the receiver D2 can be placed, D1 is located at a fixed and known position $\mathbf{q}=[0,0]^{\mathrm{T}}$, and 3 point scatterers map based-modeled [47], [48] and fixed ${ }^{5}$ at $\mathbf{s}_{1}=[5,5]^{\mathrm{T}}, \mathbf{s}_{2}=[10,-10]^{\mathrm{T}}$, and $\mathbf{s}_{3}=$ $[30,10]^{\mathrm{T}}$, respectively; hence $K=4$. We set $f_{c}=60 \mathrm{GHz}$, $B=100 \mathrm{MHz}, N_{0}=-84 \mathrm{dBm} / \mathrm{GHz}, \alpha=0 \mathrm{rad}$. For the LOS path, we set $h_{0}=\exp \left(-j 2 \pi f_{c} \tau_{0}\right) / \sqrt{\rho_{0}}$, where $\rho_{0}=$ $(2 \pi\|\mathbf{q}-\mathbf{p}\| / \lambda)^{2}$ is the path-loss between D1 and D2. For the NLOS paths, we set $h_{k}=R \exp \left(-j 2 \pi f_{c} \tau_{k}\right) / \sqrt{\rho_{k}}$, in which $\rho_{k}=\left(8 \pi\left(\left\|\mathbf{q}-\mathbf{s}_{k}\right\| \times\left\|\mathbf{s}_{k}-\mathbf{p}\right\|\right) / \lambda\right)^{2}$ [2], [47], where $R$ is the radius of the scatterer in meters. The number of antennas

${ }^{5}$ Note that the results are given for fixed positions of the scatterers to show their effect in the area. However, results for randomly placed scatterers (not shown here for space reasons) show that the PEB and SNR are relatively insensitive to the position of the scatterers and demonstrate the robustness of the positioning-based beam selection methods.

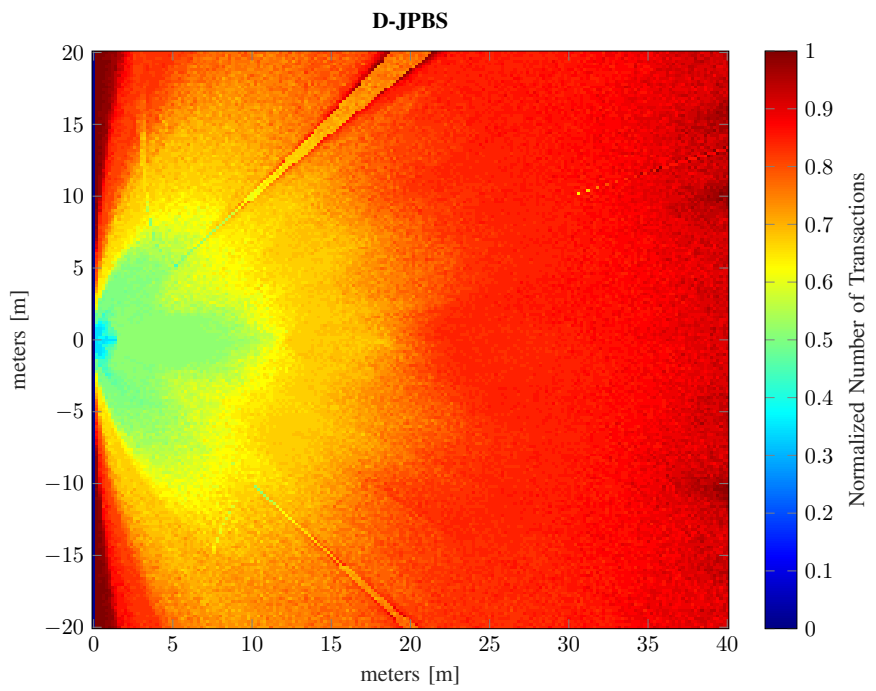

Figure 3. Normalized number of transactions with respect to the conventional beam selection protocol with fixed $N_{\text {trans }}=30$ for the discrete joint positioning and beam selection protocol.

at both D1 and D2 is $N_{t}=N_{r}=64$, and the inter-element spacing is $d=\lambda / 2$. The ULAs are located along the vertical axis. We generate a signal $x(t)$ with $N=64$ symbols. We set remaining parameters such that the SNR given by (8) on the horizontal axis at 10 meters from $\mathrm{D} 1$ is $0 \mathrm{~dB}$ (i.e., the nominal communication range is $10 \mathrm{~m}$ ).

We will evaluate three protocols in terms of SNR, number of transactions, and positioning quality (using $\mathrm{PEB}^{(I)}$ and $\left.\operatorname{REB}^{(I)}\right)$ :

- A conventional beam selection protocol (termed CBS), similar to [9], which can use only $M_{t} \in\{2,4,8,16,32,64\}$ discrete beams and $N_{t}^{\prime}=M_{t}$ active contiguous antennas sequentially selected, generated using phase shifters $\phi_{i}=\{\pi,-\pi, \pi / 2,-\pi / 2\}$. For the sake of completeness, we include out-of-band positioning (OBP) information for the conventional algorithm modeled as a Gaussian approximation $\hat{\mathbf{p} \text { out }} \sim \mathcal{N}(\mathbf{p}, \boldsymbol{\Sigma})$, where $\mathbf{p}$ is the position of the receiver D1 and $\boldsymbol{\Sigma}=\operatorname{diag}\left(\sigma_{\text {out }}^{2}, \sigma_{\text {out }}^{2}\right)$, where we set $\sigma_{\text {out }}^{2}=20 \mathrm{~m}$. The position estimate serves as extra information to reduce the set up time in the conventional beam selection approach by skipping initial coarse levels in the conventional protocol using a similar approach as in (20). We note that the OBP information does not provide at new estimate of the position at every new iteration.

- A discretized joint positioning and beam selection protocol (termed D-JPBS), using the same discrete codebook as the conventional beam selection protocol.

- A joint positioning and beam selection protocol (termed C-JPBS), with a continuous codebook of the form (6), where $\theta_{m} \in[-\pi / 2, \pi / 2]$ and $N_{t}^{\prime} \in[2,64]$.

Both positioning beam selection protocols, D-JPBS and CJPBS, generate estimates of the position of D1 as $\hat{\mathbf{p}} \sim$ $\mathcal{N}\left(\mathbf{p},\left[\mathbf{J}_{\boldsymbol{\eta}^{\prime}}^{(i)}\right]_{1: 2,1: 2}^{-1}\right)$ where $[\cdot]_{1: 2,1: 2}^{-1}$ denotes the $2 \times 2$ upper left submatrix of the inverse of the argument. The Fisher information matrix is computed using (14). 


\section{B. Results and Discussion}

We show results in two forms: contour plots over the area showing average performance (over 30 realizations) for the D-JPBS and plots that show the performance as a function of distance, where we averaged the values of the contour plots along concentric circles around D1.

1) Final SNR: Figure 2 shows the SNR as a function of the D1-D2 distance for the CBS, C-JPBS, and D-JPBS, respectively. Overall, all protocols show a similar performance in terms of SNR. The CBS (with and without OBP information, hence only CBS is shown in Figure 2) and D-JPBS protocols show identical performance. We can observe that the C-JPBS protocol achieves a slightly higher SNR with increasing distance between devices. The higher SNR is due to the higher degree of freedom that the C-JPBS protocol has compared to the other two protocols, and which allows the C-JPBS protocol to point the beams directly to the position of D2. In contrast, the CBS and D-JPBS protocols employ a more restricted codebook, and hence the SNR is dependent on the discretization of the beams. We conclude that the positioningbased protocols have no significant negative impact on the final SNR.

2) Number of transactions: The CBS uses a fixed number of transactions to complete the procedure regardless of D2 location. For this particular simulation setup the fixed transactions for the CBS protocol result in $N_{\text {trans }}=30$. Hence, we show the contour plot of the normalized number of transactions with respect to the fixed CBS transactions in Figure 3 for the D-JPBS protocol, as an example of the behavior of the protocol in terms of transactions. It can be observed that number of transactions is dependent on the discretization of the beams. We note distinct regions in the figure due to discrete number of antennas that can be used by the protocol, combined with the criterion (20). Since the beams are wider at the endfire of the D1 array, more transactions are used in the upper and lower left regions of the areas. Moreover, behind the scatterer we can observe a peculiar behavior caused by the inability of D1 in such locations to estimate both its own location and the scatterer location. In particular, the paths within this region are resolvable in angle but not in delay, creating the need for more transactions. Figure 4 shows the number of transactions as a function of D1-D2 distance. We observe that for the CBS with out-of-band position information becomes faster as a function of distance. The uncertainty in the position is easier to cover with codebook beams at a large distance. Hence, reducing the number of transactions as compared to the CBS. As distance grows larger, the number of transactions for both the D-JPBS and C-JPBS protocols increases, since we need more information in the FIM to jump to a higher number of contiguous active antennas. We can observe a reduction of $67 \%$ in the number of transactions is achieved when D2 is close to D1 (3 meters or less). The reduction grows to $50 \%$ at inter-device distances between 3 and 7 meters. Beyond 7 meters we start observing a gap between the D-JPBS and C-JPBS protocols. This is due to the codebook restriction in the D-JPBS. The beam discretization has more influence at larger distances, given the separation

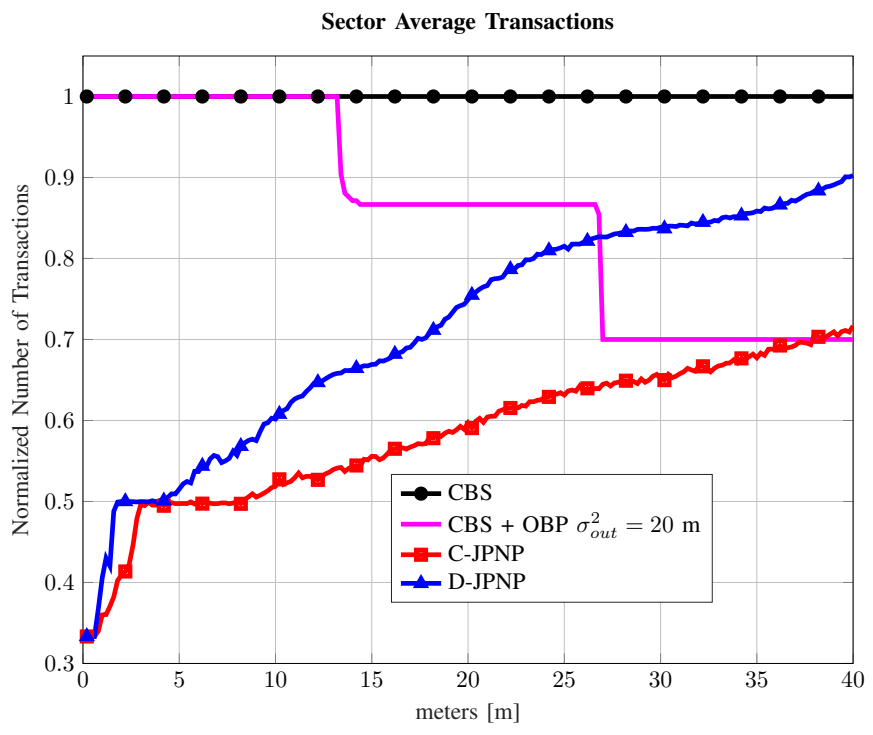

Figure 4. Average number of normalized transactions with respect to the conventional beam selection protocol with fixed $N_{\text {trans }}=30$, as a function of distance to D1.

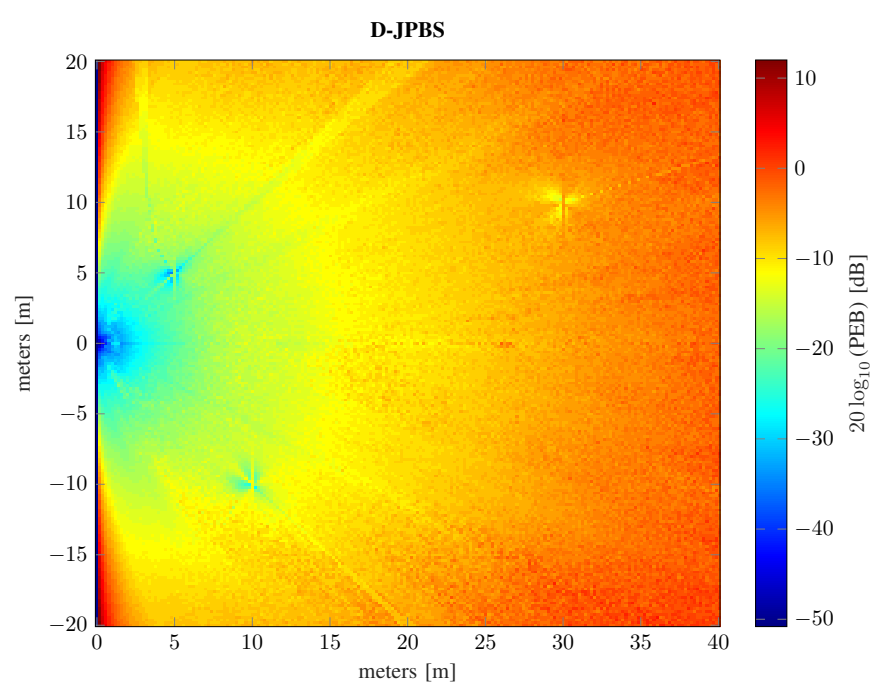

Figure 5. PEB for the discrete joint positioning and beam selection protocol. Note that a PEB of $0 \mathrm{~dB}$ corresponds to an uncertainty of $1 \mathrm{~m}, 10 \mathrm{~dB}$ corresponds to $3.2 \mathrm{~m}$, and $-10 \mathrm{~dB}$ to $30 \mathrm{~cm}$.

between the beams; thus, giving an advantage to the C-JPBS protocol which has no codebook restrictions and beams can be pointed at any direction. We can conclude that position information has an impact in the reduction of latency of the device-to-device beam selection protocol.

3) Positioning performance: Figure 5 shows the PEB for the D-JPBS. Note that a PEB of $0 \mathrm{~dB}$ corresponds to an uncertainty of $1 \mathrm{~m}, 10 \mathrm{~dB}$ corresponds to $3.2 \mathrm{~m}$, and $10 \mathrm{~dB}$ to $30 \mathrm{~cm}$. The achieved PEB values depend on the choice of the number of symbols $N$. As expected, the PEB values also depend on the distance and AOD with respect to D2. Due to high SNR gains, very low PEB values are observed close to D1. Moreover, in the regions around the position of the scatterers $\mathbf{s}_{1}=[5,5]^{\mathrm{T}}, \mathbf{s}_{2}=[10,-10]^{\mathrm{T}}$, and $\mathbf{s}_{3}=[30,10]^{\mathrm{T}}$ we can observe a small decrease in PEB due to 


$$
\mathbf{J}_{(i, j)}=\left[\begin{array}{ccccc}
\Phi\left(\tau_{i}, \tau_{j}\right) & \Phi\left(\tau_{i}, \theta_{\mathrm{tx}, j}\right) & \Phi\left(\tau_{i}, \theta_{\mathrm{rx}, j}\right) & \Phi\left(\tau_{i}, h_{R, j}\right) & \Phi\left(\tau_{i}, h_{I, j}\right) \\
\Phi\left(\theta_{\mathrm{tx}, i}, \tau_{j}\right) & \Phi\left(\theta_{\mathrm{tx}, i}, \theta_{\mathrm{tx}, j}\right) & \Phi\left(\theta_{\mathrm{tx}, i}, \theta_{\mathrm{rx}, j}\right) & \Phi\left(\theta_{\mathrm{tx}, i}, h_{R, j}\right) & \Phi\left(\theta_{\mathrm{tx}, i}, h_{I, j}\right) \\
\Phi\left(\theta_{\mathrm{rx}, i}, \tau_{j}\right) & \Phi\left(\theta_{\mathrm{rx}, i}, \theta_{\mathrm{tx}, j}\right) & \Phi\left(\theta_{\mathrm{rx}, i}, \theta_{\mathrm{rx}, j}\right) & \Phi\left(\theta_{\mathrm{rx}, i}, h_{R, j}\right) & \Phi\left(\theta_{\mathrm{rx}, i}, h_{I, j}\right) \\
\Phi\left(h_{R, i}, \tau_{j}\right) & \Phi\left(h_{R, i}, \theta_{\mathrm{tx}, j}\right) & \Phi\left(h_{R, i}, \theta_{\mathrm{rx}, j}\right) & \Phi\left(h_{R, i}, h_{R, j}\right) & \Phi\left(h_{R, i}, h_{I, j}\right) \\
\Phi\left(h_{I, i}, \tau_{j}\right) & \Phi\left(h_{I, i}, \theta_{\mathrm{tx}, j}\right) & \Phi\left(h_{I, i}, \theta_{\mathrm{rx}, j}\right) & \Phi\left(h_{I, i}, h_{R, j}\right) & \Phi\left(h_{I, i}, h_{I, j}\right)
\end{array}\right]
$$

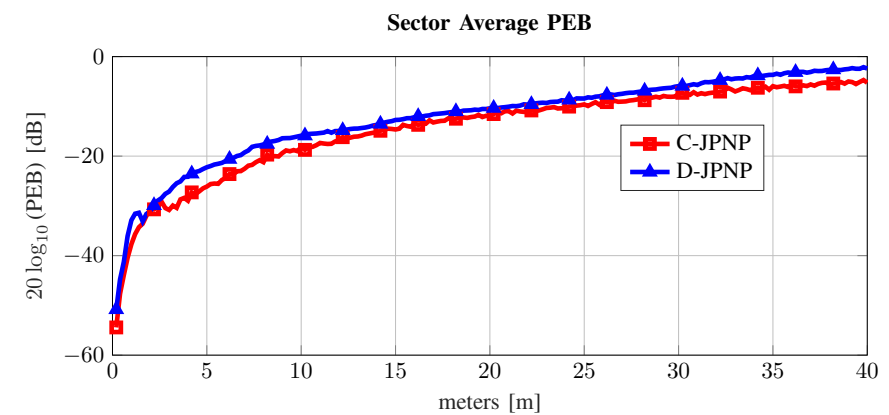

Figure 6. Average PEB as a function of distance to D1. A PEB of $0 \mathrm{~dB}$ corresponds to an uncertainty of $1 \mathrm{~m}, 10 \mathrm{~dB}$ corresponds to $3.2 \mathrm{~m}$, and -10 $\mathrm{dB}$ to $30 \mathrm{~cm}$.

the aggregate information to the FIM provided by the scatterer. This behavior is only apparent close to the scatterer given our path loss model for the scattered path. The discretized protocol shows more accurate PEB in the directions of the available beams within the codebook. We note that within the region behind the scatterer there is a decrease in PEB due to the poor resolvability of the paths and thus the inability of D2 to estimate both its own location and the scatterer location. The paths for this region are not resolvable in time, but are resolvable in the angle domain. This translates into poor delay information, which causes a degradation of the estimated parameters and hence of the PEB.

Figure 6 shows the PEB as a function the D1-D2 distance. The general trend is that the PEB increases as a function of distance, but we can observe a change around 3 meters, where the PEB abruptly decreases due to the increase in number of transactions, which provide more information to the FIM. Similar behavior is observed for the REB, hence only the PEB figures are presented. We can observe how accurate position information is attainable within a moderate distance between devices.

\section{CONCLUSIONS}

Beam selection is an essential step in establishing a mmwave communication link. Conventional protocols rely on measuring the received power obtained with a set of beamformers, which are successively made more and more directive. Given the characteristics of $\mathrm{mm}$-wave propagation and the use of standard geometric channel models, we have exploited the ability of the receiver to determine its location during the beam selection process and thus improve the subsequent selection of beams. We have shown that such in-band position-aided protocols have similar performance as the conventional protocol in terms of achieved final SNR, but they are significantly faster and can additionally provide the position or orientation of the device in an accurate manner. Such information can be used in other procedures or applications such as transmission of control. Our analysis indicates that standard codebooks can be used to harness these gains, with similar performance to more complex codebooks. Future work will include the removal of assumptions in the idealized receiver, imperfections both at the transmitter and receiver, the introduction of beamforming at the receiver and performance evaluation in complex scenarios including reflecting surfaces, point scatterers and diffuse scattering.

\section{APPENDIX A}

\section{DERIVATION OF THE FIM}

We consider the case of multiple paths and a single beam. The general form of the FIM for $k$ paths is given by

$$
\mathbf{J}_{\boldsymbol{\eta}}^{\text {(beam })}=\left[\begin{array}{ccc}
\mathbf{J}_{(1, k)} & \cdots & \mathbf{J}_{(1, k)} \\
\vdots & \ddots & \vdots \\
\mathbf{J}_{(k, 1)} & \cdots & \mathbf{J}_{(k, k)}
\end{array}\right]
$$

where each of the sub-matrices has the form (21), in which [44]

$\Phi\left(x_{1}, x_{2}\right)=\mathbb{E}_{\mathbf{y}, \mathbf{g} \mid \boldsymbol{\eta}}\left\{\left(\frac{\partial}{\partial x_{1}} \Lambda(\mathbf{y} \mid \boldsymbol{\eta}, \mathbf{g})\right)\left(\frac{\partial}{\partial x_{2}} \Lambda(\mathbf{y} \mid \boldsymbol{\eta}, \mathbf{g})\right)\right\}$,

where we recall the unknown vector parameter

$$
\boldsymbol{\eta}=\left[\tau_{0}, \boldsymbol{\theta}_{0}^{\mathrm{T}}, \mathbf{h}_{0}^{\mathrm{T}}, \ldots, \tau_{K-1}, \boldsymbol{\theta}_{K-1}^{\mathrm{T}}, \mathbf{h}_{K-1}^{\mathrm{T}}\right]^{\mathrm{T}}
$$

and $\mathrm{g}$ denotes the transmitted known training symbols. The log-likelihood function is expressed as [44]

$$
\Lambda(\mathbf{y} \mid \boldsymbol{\eta}, \mathbf{g})=-\frac{1}{N_{0}} \int\left\|\mathbf{y}(t)-\sum_{k=0}^{K-1} \mathbf{H}_{k} \mathbf{f} x\left(t-\tau_{k}\right)\right\|^{2} \mathrm{~d} t
$$

Denoting the noise-free signal by

$$
\mathbf{m}(t)=\sum_{k=0}^{K-1} \mathbf{H}_{k} \mathbf{f} x\left(t-\tau_{k}\right)
$$

it can be shown that [44, Eq. 3.33]

$$
\Phi\left(x_{1}, x_{2}\right)=\frac{2}{N_{0}} \Re\left\{\mathbb{E}_{\mathbf{g}}\left\{\int \frac{\partial \mathbf{m}^{\mathrm{H}}(t)}{\partial x_{1}} \frac{\partial \mathbf{m}(t)}{\partial x_{2}} \mathrm{~d} t\right\}\right\} .
$$


It is readily verified that for an arbitrary path $i$

$$
\begin{aligned}
\frac{\partial \mathbf{m}(t)}{\partial \tau_{i}} & =-\sqrt{N_{t} N_{r}} h_{i} \mathbf{a}_{\mathrm{rx}}\left(\theta_{\mathrm{rx}, i}\right) \mathbf{a}_{\mathrm{tx}}^{\mathrm{H}}\left(\theta_{\mathrm{tx}, i}\right) \mathbf{f} \dot{x}\left(t-\tau_{i}\right) \\
\frac{\partial \mathbf{m}(t)}{\partial \theta_{\mathrm{tx}, i}} & =\sqrt{N_{t} N_{r}} h_{i} \mathbf{a}_{\mathrm{rx}}\left(\theta_{\mathrm{rx}, i}\right) \dot{\mathbf{a}}_{\mathrm{tx}}^{\mathrm{H}}\left(\theta_{\mathrm{tx}, i}\right) \mathbf{f} x\left(t-\tau_{i}\right) \\
\frac{\partial \mathbf{m}(t)}{\partial \theta_{\mathrm{rx}, i}} & =\sqrt{N_{t} N_{r}} h_{i} \dot{\mathbf{a}}_{\mathrm{rx}}\left(\theta_{\mathrm{rx}, i}\right) \mathbf{a}_{\mathrm{tx}}^{\mathrm{H}}\left(\theta_{\mathrm{tx}, i}\right) \mathbf{f} x\left(t-\tau_{i}\right) \\
\frac{\partial \mathbf{m}(t)}{\partial h_{R, i}} & =\sqrt{N_{t} N_{r}} \mathbf{a}_{\mathrm{rx}}\left(\theta_{\mathrm{rx}, i}\right) \mathbf{a}_{\mathrm{tx}}^{\mathrm{H}}\left(\theta_{\mathrm{tx}, i}\right) \mathbf{f} x\left(t-\tau_{i}\right) \\
\frac{\partial \mathbf{m}(t)}{\partial h_{I, i}} & =\sqrt{N_{t} N_{r}} j \mathbf{a}_{\mathrm{rx}}\left(\theta_{\mathrm{rx}, i}\right) \mathbf{a}_{\mathrm{tx}}^{\mathrm{H}}\left(\theta_{\mathrm{tx}, i}\right) \mathbf{f} x\left(t-\tau_{i}\right) .
\end{aligned}
$$

\section{Diagonal elements of the FIM}

We easily find the diagonal elements of the submatrices $\mathbf{J}_{(i, j)}$. First, we define $1 / \sigma^{2}=2 N E_{s} N_{r} N_{t} / N_{0}$, $\gamma_{\mathrm{tx}, i}=\mathbf{f}^{\mathrm{H}} \mathbf{a}_{\mathrm{tx}}\left(\theta_{\mathrm{tx}, i}\right), \quad \beta_{i j}=\mathbf{a}_{\mathrm{rx}}^{\mathrm{H}}\left(\theta_{\mathrm{rx}, i}\right) \mathbf{a}_{\mathrm{rx}}\left(\theta_{\mathrm{rx}, j}\right)$ as well as $\dot{\mathbf{a}}_{\mathrm{tx}}\left(\theta_{\mathrm{tx}}\right)=\partial \mathbf{a}_{\mathrm{tx}}\left(\theta_{\mathrm{tx}}\right) / \partial \theta_{\mathrm{tx}}, \dot{\gamma}_{\mathrm{tx}, \mathrm{i}}=\dot{\mathbf{a}}_{\mathrm{tx}}^{\mathrm{H}}\left(\theta_{\mathrm{tx}, i}\right) \mathbf{f}$, and $\ddot{\beta}_{i j}=\dot{\mathbf{a}}_{\mathrm{rx}}^{\mathrm{H}}\left(\theta_{\mathrm{rx}, i}\right) \dot{\mathbf{a}}_{\mathrm{rx}}\left(\theta_{\mathrm{rx}, j}\right)$. Recalling $p(t)$ is a unit-energy transmit pulse, we also introduce

$$
\begin{aligned}
& A_{0}(\Delta)=\int p^{*}(t-\Delta) p(t) \mathrm{d} t \\
& A_{1}(\Delta)=\int \dot{p}^{*}(t-\Delta) p(t) \mathrm{d} t \\
& A_{2}(\Delta)=\int \dot{p}^{*}(t-\Delta) \dot{p}(t) \mathrm{d} t .
\end{aligned}
$$

We then find that

$$
\begin{aligned}
\Phi\left(\tau_{i}, \tau_{j}\right) & =\frac{1}{\sigma^{2}} \Re\left\{h_{i}^{*} h_{j} \gamma_{\mathrm{tx}, i} \gamma_{\mathrm{tx}, j}^{*} \beta_{i j} A_{2}\left(\Delta_{i j}\right)\right\}, \\
\Phi\left(\theta_{\mathrm{tx}, i}, \theta_{\mathrm{tx}, j}\right) & =\frac{1}{\sigma^{2}} \Re\left\{h_{i}^{*} h_{j} \dot{\gamma}_{\mathrm{tx}, i}^{*} \beta_{i j} \dot{\gamma}_{\mathrm{tx}, j} A_{0}\left(\Delta_{i j}\right)\right\} \\
\Phi\left(\theta_{\mathrm{rx}, i}, \theta_{\mathrm{rx}, j}\right) & =\frac{1}{\sigma^{2}} \Re\left\{h_{i}^{*} h_{j} \gamma_{\mathrm{tx}, i} \ddot{\beta}_{i j} \gamma_{\mathrm{tx}, j}^{*} A_{0}\left(\Delta_{i j}\right)\right\} \\
\Phi\left(h_{R, i}, h_{R, j}\right) & =\Phi\left(h_{I, i}, h_{I, j}\right)=\frac{1}{\sigma^{2}} \Re\left\{\gamma_{\mathrm{tx}, i} \gamma_{\mathrm{tx}, j}^{*} \beta_{i j} A_{0}\left(\Delta_{i j}\right)\right\}
\end{aligned}
$$

where $\Delta_{i j}=\tau_{i}-\tau_{j}$.

\section{Off-diagonal elements of the FIM}

The off-diagonal elements are computed in similar fashion. Introducing, $\dot{\beta}_{i j}=\mathbf{a}_{\mathrm{rx}}^{\mathrm{H}}\left(\theta_{\mathrm{rx}, i}\right) \dot{\mathbf{a}}_{\mathrm{rx}}\left(\theta_{\mathrm{rx}, j}\right)$, the final expressions for the upper diagonal elements are computed as:

$$
\begin{aligned}
\Phi\left(\tau_{i}, \theta_{\mathrm{tx}, j}\right) & =-\frac{1}{\sigma^{2}} \Re\left\{h_{i}^{*} h_{j} \gamma_{\mathrm{tx}, i} \beta_{i j} \dot{\gamma}_{\mathrm{tx}, j} A_{1}\left(\Delta_{i j}\right)\right\} \\
\Phi\left(\tau_{i}, \theta_{\mathrm{rx}, j}\right) & =-\frac{1}{\sigma^{2}} \Re\left\{h_{i}^{*} h_{j} \gamma_{\mathrm{tx}, i} \dot{\beta}_{i j} \gamma_{\mathrm{tx}, j}^{*} A_{1}\left(\Delta_{i j}\right)\right\} \\
\Phi\left(\tau_{i}, h_{R, j}\right) & =-\frac{1}{\sigma^{2}} \Re\left\{h_{i}^{*} \gamma_{\mathrm{tx}, i} \beta_{i j} \gamma_{\mathrm{tx}, j}^{*} A_{1}\left(\Delta_{i j}\right)\right\} \\
\Phi\left(\tau_{i}, h_{I, j}\right) & =-\frac{1}{\sigma^{2}} \Re\left\{j h_{i}^{*} \gamma_{\mathrm{tx}, i} \beta_{i j} \gamma_{\mathrm{tx}, j}^{*} A_{1}\left(\Delta_{i j}\right)\right\} \\
\Phi\left(\theta_{\mathrm{tx}, i}, \theta_{\mathrm{rx}, j}\right) & =\frac{1}{\sigma^{2}} \Re\left\{h_{i}^{*} h_{j} \dot{\gamma}_{\mathrm{tx}, i}^{*} \dot{\beta}_{i j} \gamma_{\mathrm{tx}, j}^{*} A_{0}\left(\Delta_{i j}\right)\right\} \\
\Phi\left(\theta_{\mathrm{tx}, i}, h_{R, j}\right) & =\frac{1}{\sigma^{2}} \Re\left\{h_{i}^{*} \dot{\gamma}_{\mathrm{tx}, i}^{*} \beta_{i j} \gamma_{\mathrm{tx}, j}^{*} A_{0}\left(\Delta_{i j}\right)\right\} \\
\Phi\left(\theta_{\mathrm{tx}, i}, h_{I, j}\right) & =\frac{1}{\sigma^{2}} \Re\left\{j h_{i}^{*} \dot{\gamma}_{\mathrm{tx}, i}^{*} \beta_{i j} \gamma_{\mathrm{tx}, j}^{*} A_{0}\left(\Delta_{i j}\right)\right\} \\
\Phi\left(\theta_{\mathrm{rx}, i}, h_{R, j}\right) & =\frac{1}{\sigma^{2}} \Re\left\{h_{i}^{*} \gamma_{\mathrm{tx}, i} \dot{\beta}_{j i}^{*} \gamma_{\mathrm{tx}, j}^{*} A_{0}\left(\Delta_{i j}\right)\right\} \\
\Phi\left(\theta_{\mathrm{rx}, i}, h_{I, j}\right) & =\frac{1}{\sigma^{2}} \Re\left\{j h_{i}^{*} \gamma_{\mathrm{tx}, i} \dot{\beta}_{i j}^{*} \gamma_{\mathrm{tx}, j}^{*} A_{0}\left(\Delta_{i j}\right)\right\} \\
\Phi\left(h_{R, i}, h_{I, i}\right) & =\frac{1}{\sigma^{2}} \Re\left\{j \gamma_{\mathrm{tx}, i} \beta_{i j} \gamma_{\mathrm{tx}, j}^{*} A_{0}\left(\Delta_{i j}\right)\right\} .
\end{aligned}
$$

The elements of the lower off diagonal are obtained as

$$
\begin{aligned}
\Phi\left(\theta_{\mathrm{tx}, i}, \tau_{j}\right) & =-\frac{1}{\sigma^{2}} \Re\left\{h_{i}^{*} h_{j} \dot{\gamma}_{\mathrm{tx}, i}^{*} \beta_{i j} \gamma_{\mathrm{tx}, j}^{*} A_{1}\left(\Delta_{j i}\right)\right\} \\
\Phi\left(\theta_{\mathrm{rx}, i}, \tau_{j}\right) & =-\frac{1}{\sigma^{2}} \Re\left\{h_{i}^{*} h_{j} \gamma_{\mathrm{tx}, i} \dot{\beta}_{j i}^{*} \gamma_{\mathrm{tx}, j}^{*} A_{1}\left(\Delta_{j i}\right)\right\} \\
\Phi\left(h_{R, i}, \tau_{j}\right) & =-\frac{1}{\sigma^{2}} \Re\left\{h_{j} \gamma_{\mathrm{tx}, i} \beta_{i j} \gamma_{\mathrm{tx}, j}^{*} A_{1}\left(\Delta_{j i}\right)\right\} \\
\Phi\left(h_{I, i}, \tau_{j}\right) & =-\frac{1}{\sigma^{2}} \Re\left\{j h_{j} \gamma_{\mathrm{tx}, i} \beta_{i j} \gamma_{\mathrm{tx}, j}^{*} A_{1}\left(\Delta_{j i}\right)\right\} \\
\Phi\left(\theta_{\mathrm{rx}, i}, \theta_{\mathrm{tx}, j}\right) & =\frac{1}{\sigma^{2}} \Re\left\{h_{i}^{*} h_{j} \gamma_{\mathrm{tx}, i} \dot{\beta}_{j i}^{*} \dot{\gamma}_{\mathrm{tx}, j} A_{0}\left(\Delta_{i j}\right)\right\} \\
\Phi\left(h_{R, i}, \theta_{\mathrm{tx}, j}\right) & =\frac{1}{\sigma^{2}} \Re\left\{h_{j} \gamma_{\mathrm{tx}, i} \beta_{i j} \dot{\gamma}_{\mathrm{tx}, j} A_{0}\left(\Delta_{i j}\right)\right\} \\
\Phi\left(h_{I, i}, \theta_{\mathrm{tx}, j},\right) & =\frac{1}{\sigma^{2}} \Re\left\{j h_{j} \gamma_{\mathrm{tx}, i} \beta_{i j} \dot{\gamma}_{\mathrm{tx}, j} A_{0}\left(\Delta_{i j}\right)\right\} \\
\Phi\left(h_{R, i}, \theta_{\mathrm{rx}, j}\right) & =\frac{1}{\sigma^{2}} \Re\left\{h_{j} \gamma_{\mathrm{tx}, i} \dot{\beta}_{i j} \gamma_{\mathrm{tx}, j}^{*} A_{0}\left(\Delta_{i j}\right)\right\} \\
\Phi\left(h_{I, i}, \theta_{\mathrm{rx}, j}\right) & =\frac{1}{\sigma^{2}} \Re\left\{j h_{j} \gamma_{\mathrm{tx}, i} \dot{\beta}_{i j} \gamma_{\mathrm{tx}, j}^{*} A_{0}\left(\Delta_{i j}\right)\right\} \\
\Phi\left(h_{I, i}, h_{R, j}\right) & =\frac{1}{\sigma^{2}} \Re\left\{j \gamma_{\mathrm{tx}, i} \beta_{i j} \gamma_{\mathrm{tx}, j}^{*} A_{0}\left(\Delta_{i j}\right)\right\}
\end{aligned}
$$

Remarks:

- When $p(t)$ is flat in the frequency domain, then

$$
\begin{aligned}
A_{0}(\Delta) & =\frac{\sin (\pi B \Delta)}{\pi B \Delta} \\
A_{1}(\Delta) & =\frac{-\sin (\pi B \Delta)+\pi B \Delta \cos (\pi B \Delta)}{\pi B \Delta^{2}} . \\
A_{2}(\Delta) & =\frac{\left((\pi B \Delta)^{2}-2\right) \sin (\pi B \Delta)}{\pi \Delta^{3} B} \\
& +\frac{2 \pi B \Delta \cos (\pi B \Delta)}{\pi \Delta^{3} B}
\end{aligned}
$$

We observe that $A_{0}(0)=1, A_{1}(0)=0$, and $A_{2}(0)=$ $\pi^{2} B^{2} / 3$, so that the entries in $\mathbf{J}_{(i, i)}$ have compact expressions, compared to $\mathbf{J}_{(i, j \neq i)}$. 
- When $\Delta$ is such that $B \Delta \gg 1$, then $A_{0,1,2}(\Delta) \approx 0$. Hence, when paths have large relative path lengths, this leads to a block diagonal structure in (22).

\section{APPENDIX B \\ OPTIMIZED BEAM DiRECTIONS}

The directions of the beams in the position-aided protocol can be optimized as follows. We select one beam with maximum gain direction $\theta_{0}$ closest to $\hat{\theta}_{\mathrm{tx}, 0}^{(i)}$. Then, the two additional beams are set to minimize a measure of expected future uncertainty. Let $\mathbf{J}_{\hat{\boldsymbol{\eta}}}^{(i, m)}(\theta)$ be the FIM, evaluated in $\hat{\boldsymbol{\eta}}$ (the estimate of $\boldsymbol{\eta}$ ) for a beam pointing towards $\theta$. The we choose beams

$$
\begin{aligned}
\operatorname{minimize}_{\theta_{1}, \theta_{2}} & \operatorname{trace}\left\{\left[\mathbf{J}_{\hat{\boldsymbol{\eta}}}^{(i, 1)}\left(\theta_{0}\right)\right.\right. \\
& \left.\left.+\mathbf{J}_{\hat{\boldsymbol{\eta}}}^{(i, 2)}\left(\theta_{1}\right)+\mathbf{J}_{\hat{\boldsymbol{\eta}}}^{(i, 3)}\left(\theta_{2}\right)\right]_{1: 2,1: 2}^{-1}\right\}
\end{aligned}
$$

subject to $\theta_{1}, \theta_{2} \in \Theta$.

In case $\Theta$ is $[-\pi / 2, \pi / 2]$, we can instead set $\theta_{0}=\hat{\theta}_{\mathrm{tx}, 0}^{(i)}$, $\theta_{1}=\hat{\theta}_{\mathrm{tx}, 0}^{(i)}+\varepsilon$ and $\theta_{2}=\hat{\theta}_{\mathrm{tx}, 0}^{(i)}-\varepsilon$, and optimize with respect to the scalar parameter $\varepsilon \geq 0$.

\section{REFERENCES}

[1] P. Zhouyue and F. Khan, "An introduction to millimeter-wave mobile broadband systems," IEEE Communications Magazine, vol. 49, no. 6, pp. 101-107, 2011.

[2] T. Rappaport, S. Sun, R. Mayzus, H. Zhao, Y. Azar, K. Wang, G. Wong, J. Schulz, M. Samimi, and F. Gutierrez, "Millimeter wave mobile communications for 5G cellular: It will work!" IEEE Access, vol. 1, pp. 335-349, 2013.

[3] A. L. Swindlehurst, E. Ayanoglu, P. Heydari, and F. Capolino, "Millimeter-wave massive MIMO: The next wireless revolution?" IEEE Communications Magazine, pp. 56-62, 2014.

[4] S. Sun, R. Theodore S. Rappaport andand W. Heath Jr., A. Nix, and S. Rangan, "MIMO for millimeter-wave wireless communications: beamforming, spatial multiplexing, or both?" IEEE Communications Magazine, vol. 52, pp. 110-121, 2014.

[5] T. Bai, A. Alkhateeb, and R. W. Heath Jr., "Coverage and capacity of millimeter-wave cellular networks," IEEE Communications Magazine, vol. 52, pp. 70-77, 2014.

[6] C. J. Hansen, "WiGiG: Multi-gigabit wireless communications in the 60 GHz band," IEEE Wireless Communications, vol. 18, 2011.

[7] K. Venugopal and R. W. Heath Jr., "Millimeter wave networked wearables in dense indoor environments," IEEE Access, 2016.

[8] J. Choi, V. Va, N. González-Prelcic, R. Daniels, C. R. Bhat, , and R. W. Heath Jr., "Millimeter-wave vehicular communication to support massive automotive sensing," IEEE Communications Magazine, vol. 54, pp. 160-167, 2016.

[9] J. Wang, "Beam codebook based beamforming protocol for multi-Gbps millimeter-wave WPAN systems," IEEE Journal on Selected Areas in Communications, vol. 27, no. 8, pp. 1390-1399, 2009.

[10] S. Hur, T. Kim, D. Love, J. Krogmeier, T. Thomas, and A. Ghosh, "Millimeter wave beamforming for wireless backhaul and access in small cell networks," IEEE Transactions on Communications, vol. 61, no. 10, pp. 4391-4403, 2013.

[11] Y. M. Tsang, A. S. Y. Poon, and S. Addepalli, "Coding the beams: Improving beamforming training in mmwave communication system," in IEEE Global Communications Conference, 2011.

[12] R. J. Lopes and J. M. Brázio, "A geometry-based statistical model for fast fading in millimeterwave communications," in IEEE Vehicular Technology Conference, vol. 1, 1995, pp. 459-463.

[13] H. Deng and A. Sayeed, "Mm-wave MIMO channel modeling and user localization using sparse beamspace signatures," in International Workshop on Signal Processing Advances in Wireless Communications, 2014, pp. 130-134.
[14] Q. Li, G. Wu, , and T. S. Rappaport, "Channel model for millimeterwave communications based on geometry statistics," in IEEE Global Communications Conference Workshops, 2014.

[15] A. Alkhateeb, O. E. Ayach, G. Leus, , and R. W. Heath Jr., "Channel estimation and hybrid precoding for millimeter wave cellular systems," IEEE Journal of Selected Topics in Signal Processing, vol. 8, no. 5, pp. 831-846, 2014

[16] M. Steinbauer, A. F. Molisch, and E. Bonek, "The double-directional radio channel," IEEE Antennas and Propagations Magazine, vol. 43, pp. 51-63, 2001.

[17] H. Lee and Y. Ko, "Low complexity codebook-based beamforming for MIMO-OFDM systems in millimiter-wave WPAN," IEEE Transactions on Wireless Communications, vol. 10, pp. 3607-3612, 2011.

[18] P. Xia, S. Yong, J. Oh, and C. Ngo, "A practical SDMA protocol for $60 \mathrm{GHz}$ millimiter wave communications," in Asilomar Conference on Signals, Systems and Computers, 2008.

[19] L. Chen, Y. Yang, X. Chen, and W. Wang, "Multi-stage communications codebook for 60GHz WPAN," in 6th International Conference on Communications and Networking in China, 2011.

[20] Z. Xiao, T. He, P. Xia, and X. Xia, "Hierarchical codebook design for beamforming training in millimeter-wave communication," IEEE Transactions on Wireless Communications, vol. 15, pp. 3380 - 3392, 2016.

[21] M. Kokshoorn, H. Chen, P. Wang, Y. Li, and B. Vucetic, "Millimeter wave MIMO channel estimation using overlapped beam patterns and rate adaptation," IEEE Transactions on Signal Processing, vol. 65, no. 3, pp. 601-616, 2017.

[22] C. N. Barati, A. Hosseini, M. Mezzavilla, T. Korakis, S. S. Panwar, S. Rangan, and M. Zorzi, "Initial access in millimeter wave cellular systems," IEEE Transactions on Wireless Communications, vol. 15, pp. 7926 - 7940, 2016.

[23] C. Liu, M. Li, I. B. Collings, S. V. Hanly, and P. Whiting, "Design and analysis of transmit beamforming for millimeter wave base station discovery," IEEE Transactions on Wireless Communications, vol. 16, pp. 797-811, 2017.

[24] J. Kim and A. F. Molisch, "Fast millimeter-wave beam training with receive beamforming," Journal of Communications and Networks, vol. 16, pp. 512-522, 2014.

[25] A. Hu, T. Lv, H. Gao, Z. Zhang, and S. Yang, "An ESPRIT-based approach for 2-D localization of incoherently distributed sources in massive MIMO systems," IEEE Journal of Selected Topics in Signal Processing, vol. 8, no. 5, pp. 996-1011, 2014.

[26] P. Sanchis, J. Martinez, J. Herrera, V. Polo, J. Corral, and J. Marti, "A novel simultaneous tracking and direction of arrival estimation algorithm for beam-switched base station antennas in millimeter-wave wireless broadband access networks," in IEEE Antennas and Propagation Society International Symposium, 2002.

[27] D. Zhu, J. Choi, and R. W. Heath Jr., "Auxiliary beam pair design in mmwave cellular systems with hybryd precoding and limited feedback," in International Conference on Acoustics, Speech and Signal Processing, 2016.

[28] B. Peng and T. Kürner, "Three dimensional angle of arrival estimation in dynamic indoor terahertz channels using forward-backward algorithm," IEEE Transactions on Vehicular Technology (Accepted), 2016.

[29] A. Shamansoori, G. E. Garcia, G. Destino, G. Seco-Granados, and $\mathrm{H}$. Wymeersch, "5G position and orientation estimation through millimiter wave MIMO," in IEEE Global Communications Conference Workshops, 2015.

[30] G. C. Alexandropoulos, "Position aided beam alignment for millimeter wave backhaul systems with large phased arrays," 2017. [Online]. Available: http://arxiv.org/abs/1701.03291

[31] J. C. Avilés and A. Kouki, "Position-aided mm-wave beam training under NLOS conditions," IEEE Access, vol. 4, pp. 8703-8714, 2016.

[32] N. Garcia, H. Wymeersch, E. G. Strom, and D. Slock, "Locationaided mm-wave channel estimation for vehicular communication," in IEEE International workshop on Signal Processing Advances in Wireless Communication, 2016.

[33] V. Va, X. Zhang, and R. W. Heath Jr., "Beam switching for millimeter wave communication to support high speed trains," IEEE 82nd Vehicular Technology Conference (VTC Fall), 2015.

[34] K. Gao, M. Cai, D. Nie, and K. Liu, "Beampattern-based tracking for millimeter wave communication systems," in IEEE Global Communications Conference, 2016.

[35] J. Bae, S. H. Lim, J. H. Yoo, and J. W. Choi, "New beam tracking technique for millimeter wave-band communications," 2017. [Online]. Available: https://arxiv.org/abs/1702.00276v1 
[36] P. Kela, M. Costa, J. Turkka, M. Koivisto, J. Werner, A. Hakkarainen, M. Valkama, R. Jäntti, and K. Leppönen, "Location based beamforming in 5G ultra-dense networks," IEEE Vehicular Technology Conference, 2016.

[37] A. M. Sayeed, "Deconstructing multiantenna fading channels," IEEE Transactions on Signal Processing, vol. 50, pp. 2563-2579, 2002.

[38] J. Brady, N. Behdad, and A. Sayeed, "Beamspace MIMO for millimeterwave communications: System architecture, modeling, analysis, and measurements," IEEE Transactions on Antennas and Propagation, vol. 61 , no. 7, pp. 3814-3827, 2013.

[39] D. Tse and P. Viswanath, Fundamentals of Wireless Communication. Cambridge Press University, 2005.

[40] N. Celik, W. Kim, M. F. Demirkol, M. F. Iskander, and R. Emrick, "Implementation and experimental verification of hybrid smart-antenna beamforming algorithm," IEEE Antennas and Wireless Propagation Letters, vol. 5, pp. 280-283, 2006.

[41] S. J. Orfandis, Electromagnetic Waves and Antennas. Rutgers University, 2008.

[42] Y. Shen and M. Z. Win, "Fundamental limits of wideband localization part I: A general framework," IEEE Transactions on Information Theory, vol. 56, pp. 4956-4980, 2010.

[43] J. Palacios, D. de Donno, and J. Widmer, "Tracking mm-wave channel dynamics: Fast beam training strategies under mobility," IEEE Conference in Computer Communications, 2017.

[44] S. M. Kay, Fundamentals of Statistical Signal Processing: Estimation Theory. New York, NY, USA: Prentice Hall, 2010.

[45] A. Shahmansoori, G. E. García, G. Destino, G. Seco-Granados, and $\mathrm{H}$. Wymeersch, "Position and orientation estimation through millimeter wave MIMO in 5G systems," Submitted to IEEE Transactions on Wireless Communications, 2017. [Online]. Available: http://arxiv.org/abs/1702.01605

[46] A. Guerra, F. Guidi, and D. Dardari, "Position and orientation error bound for wideband massive antenna arrays," in International Conference on Communications Workshops, 2015.

[47] 3GPP, "5G: Study on channel model for frequencies from 0.5 to 100 GHz," 3GPP TR 38.901 Version 14.1.1 Release 14, Tech. Rep., 2017.

[48] V. Nurmela, A. Karttunen, A. Roivainen, L. Raschkowski, T. Imai, J. Järveläinen, J. Medbo, J. Vihriälä, J. Meinilä, K. Haneda, V. Hovinen, J. Ylitalo, N. Omaki, K. Kusume, P. Kyösti, T. Jämsä, A. Hekkala, R. Weiler, and M. Peter, "METIS channel models," Mobile and wireless communications Enablers for the Twenty-twenty Information Society (METIS), D1.4, 2015.

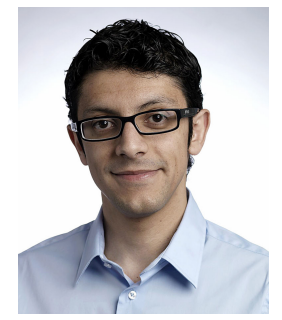

Gabriel E. Garcia (S'13) received the B.Sc. degree in electronics and communication engineering from Instituto Tecnológico y de Estudios Superiores de Monterrey (ITESM), Campus Querétaro, Mexico, in 2007, the M.Sc. degree in communication engineering, and Licentiate degree in electrical engineering, both from Chalmers University of Technology, Göteborg, Sweden, in 2010 and 2013, respectively. Since May 2011, he is pursuing the Ph.D. degree at the Department of Electrical Engineering, Chalmers University of Technology, Göteborg, Sweden. His research interests include cooperative networks, robust radio frequency localization and navigation, UWB, millimeter-wave, wireless sensor networks, GNSS, statistical inference and sensor fusion.

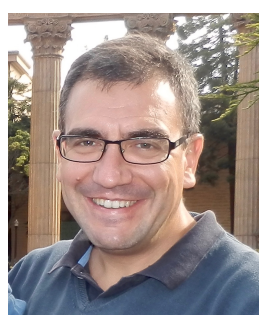

Gonzalo Seco-Granados (S'97-M'02-SM'08) received the Ph.D. degree in Telecommunications Engineering from the Universitat Politecnica de Catalunya, Spain, in 2000, and the M.B.A. degree from the IESE Business School, Spain, in 2002. From 2002 to 2005, he was a member of the European Space Agency, involved in the design of the Galileo System. Since 2006, he is with the Department of Telecommunications, Universitat Autonoma de Barcelona, where he is a Professor, and has served as Vice Dean of the Engineering School since 2011. In 2015, he was a Fulbright Visiting Professor with the University of California at Irvine, Irvine, CA, USA. His research interests include satellite and terrestrial localization systems. He was a recipient of the 2013 ICREA Academia Award. Since 2018, he has been serving as a member of the Sensor Array and Multichannel Technical Committee of the IEEE Signal Processing Society.

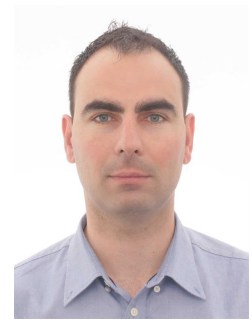

Eleftherios Karipidis (S'05?M'09) received the Diploma degree in Electrical and Computer Engineering from the Aristotle University of Thessaloniki, Greece, in 2001, the M.Sc. degree in Communications Engineering from the Technical University of Munich, Germany, in 2003, and the Ph.D. degree in Electronic and Computer Engineering from the Technical University of Crete, Chania, Greece, in 2008. From 2008 to 2013, he was a postdoctoral researcher with Communication Systems Division, Department of Electrical Engineering, Linköping University, Sweden. In June 2013, he joined Ericsson Research, Stockholm, Sweden, where he is currently a Senior Researcher, involved in the 3GPP standardization of 5G New Radio (NR). His current research interests include multi-antenna technologies and reference signal design. Since 2016, he has been serving as a member of the Signal Processing for Communications and Networking Technical Committee of the IEEE Signal Processing Society.

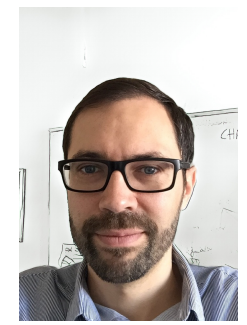

Henk Wymeersch (S'01-M'05) obtained the Ph.D. degree in Electrical Engineering/Applied Sciences in 2005 from Ghent University, Belgium. He is currently a Professor of Communication Systems with the Department of Electrical Engineering at Chalmers University of Technology, Sweden. Prior to joining Chalmers, he was a postdoctoral researcher from 2005 until 2009 with the Laboratory for Information and Decision Systems at the Massachusetts Institute of Technology. Prof. Wymeersch served as Associate Editor for IEEE Communication Letters (2009-2013), IEEE Transactions on Wireless Communications (since 2013), and IEEE Transactions on Communications (2016-2018). His current research interests include cooperative systems and intelligent transportation. 